\title{
Avaliação da capacidade funcional e prevalência de sintomas depressivos em idosos institucionalizados
}

\author{
Evaluation of functional capacity and prevalence of depressive symptoms in \\ institutionalized elderly
}

\section{Evaluación de la capacidad funcional y prevalencia de síntomas depresivos en ancianos institucionalizados}

\begin{abstract}
Keity Cristina Bueno Perina ${ }^{1 *}$, Ana Carolina Alves de Oliveira1, Priscila Maria Martins Machado
\end{abstract}
\section{RESUMO}

Objetivo: Avaliar a prevalência de sintomas depressivos e funcionalidade em idosos institucionalizados em uma cidade do Estado de Minas Gerais, MG. Métodos: Trata-se de um estudo descritivo transversal, com abordagem quantitativa, realizado mediante a coleta de dados pessoais, sociodemográficos, Mini Exame do Estado Mental, aplicação das escalas de depressão geriátrica em versão reduzida (EDG-15) e WHODAS 2.0, em 50 indivíduos que residem em três Instituições de Longa Permanência para Idosos na uma cidade do Estado de Minas Gerais, MG. Resultados: A média de idade foi de 77,5 anos, em uma amostra composta principalmente por mulheres (74\%). Dos 50 participantes estudados, $98 \%$ apresentaram algum grau de incapacidade funcional, sendo que a maioria, $36 \%$, apresentava incapacidade funcional moderada, e $60 \%$ dos idosos apresentavam sintomas depressivos, sendo que $48 \%$ destes eram mulheres. Conclusão: Conclui-se que a prevalência de sintomas depressivos e incapacidade funcional em idosos institucionalizados eram altas, $60 \%$ e $98 \%$, respectivamente. Entretanto, é necessário destacar que esse resultado indica a presença de sintomatologia depressiva, e não se refere ao diagnóstico de depressão. Ressalta-se que a detecção precoce dos sintomas depressivos é importante para evitar o desenvolvimento do quadro depressivo.

Palavras-chave: Idosos, Depressão, Institucionalização.

\begin{abstract}
Objective: To evaluate the prevalence of depressive symptoms and functionality in institutionalized elderly in a municipality in the state of Minas Gerais, MG. Methods: This is a cross-sectional descriptive study, with a quantitative approach, through the collection of personal, sociodemographic data, the Mini-Mental Stade Examination (MMSE) and application of the geriatric depression and WHODAS 2.0 scales, in 50 individuals residing in three Long Term Care Institutions for the Elderly in a municipality in the state of Minas Gerais, MG. The data obtained were analyzed using descriptive statistics using the IBM SPSS Statistics software. The study was approved by the Research Ethics Committee. Results: The average age was 77.5 years, ranging from 60 to 99 years, in a sample composed mainly of women (74\%). Of the 50 participants studied, $98 \%$ had some degree of functional disability, with the majority, $36 \%$, having moderate functional disability, and $60 \%$ of the elderly had depressive symptoms, $48 \%$ of whom were women. Conclusion: It is concluded that the prevalence of depressive symptoms and functional disability in institutionalized elderly people was high, $60 \%$ and $98 \%$, respectively. However, it is necessary to highlight that this result indicates the presence of depressive symptoms, and does not refer to the diagnosis of depression. It is noteworthy that the early detection of depressive symptoms is important to prevent the development of depressive symptoms, thus preventing its negative effects on the health and quality of life of these elderly people.
\end{abstract}

Keywords: Elderly, Depression, Institutionalization.

\footnotetext{
${ }^{1}$ Universidade do Estado de Minas Gerais (UEMG). Divinópolis - MG

*Email: keity.bueno@gmail.com
} 


\section{RESUMEN}

Objetivo: evaluar la prevalencia de síntomas depresivos y funcionalidad en ancianos institucionalizados en un municipio en el estado de Minas Gerais, MG. Métodos: Este es un estudio descriptivo de corte transversal, con un enfoque cuantitativo, a través de la recopilación de datos personales, sociodemográficos y la aplicación de la depresión geriátrica y las escalas WHODAS 2.0, en 50 individuos que residen en tres instituciones de atención a largo plazo para ancianos en un municipio en el estado de Minas Gerais. Los datos obtenidos se analizaron utilizando estadísticas descriptivas utilizando el software IBM SPSS Statistics. El estudio fue aprobado por el Comité de Ética en Investigación. Resultados: La edad promedio fue de 77.5 años, con un rango de 60 a 99 años, en una muestra compuesta principalmente por mujeres (74\%). De los 50 participantes estudiados, el $98 \%$ tenía algún grado de discapacidad funcional, con la mayoría, el 36\%, con discapacidad funcional moderada, y el $60 \%$ de los ancianos tenían síntomas depressivos, el $48 \%$ de los cuales eran mujeres. Conclusión: Se concluye que la prevalencia de síntomas depresivos y discapacidad funcional en personas mayores institucionalizadas fue alta, $60 \%$ y $98 \%$, respectivamente. Sin embargo, es necesario resaltar que este resultado indica la presencia de síntomas depresivos y no se refiere al diagnóstico de depresión. Es de destacar que la detección temprana de síntomas depresivos es importante para prevenir el desarrollo de síntomas depresivos, evitando así sus efectos negativos sobre la salud y la calidad de vida de estas personas mayores.

Palabras clave: Ancianos, Depresión, Institucionalización.

\section{INTRODUÇÃO}

O envelhecimento populacional é um fenômeno que ocorre mundialmente, sendo mais frequente e impactante nos países em desenvolvimento. Nos últimos anos, é possível observar um padrão acelerado no crescimento da população idosa em todo mundo (SOUZA VL, et al., 2015).

Atualmente, o Brasil apresenta muitas mudanças relacionadas ao perfil demográfico, acompanhando o cenário mundial, sendo considerado o sexto país do mundo em taxa de envelhecimento populacional, e esse crescimento brusco da população idosa ocorre por uma combinação de variáveis exclusivamente demográficas com profundas alterações sociais e culturais (MORAES EN, 2012).

Segundo o Instituto Brasileiro de Geografia e Estatística (IBGE), o número de idosos chega a 10,8\% da população brasileira. O Sul e Sudeste são as regiões do país com maior número de idosos, com $8,1 \%$ da população formada por indivíduos com 65 anos ou mais (IBGE, 2009). Acredita-se que em 2050, as crianças de 0 a 14 anos representarão $13,15 \%$, enquanto a população idosa alcançará $22,71 \%$ da população total, o que caracteriza a inversão da pirâmide populacional (IBGE, 2008).

Esta transição demográfica influencia na saúde por meio de mudanças no perfil de morbidade e mortalidade da população, levando à necessidade de reorganização dos modelos assistenciais. Assim, é necessário que o atendimento à saúde do idoso seja voltado para a prevenção e identificação de sinais e sintomas característicos do envelhecimento (ALVES DB e BARBOSA MTS, 2010).

O envelhecimento é um acontecimento natural, fisiológico, gradual e irreversível, acompanhado por várias alterações funcionais, com alterações psicológicas e motoras, além do aumento na prevalência de doenças (MACIEL MG, 2010; SOUZA VL, et al., 2015). No Brasil, esse processo vem ocorrendo em alta velocidade e o Sistema de Saúde ainda não conseguiu adaptar-se de forma a gerar qualidade de vida ou atender a população idosa (CARVALHO JAM e RODRÍGUEZ-WONG LL, 2008).

Considerando esse aumento no número de idosos e a longevidade da população, somados às dificuldades socioeconômicas dos idosos e seus familiares, a ausência de cuidadores para esses idosos, os conflitos familiares, cresce a demanda por Instituições de Longa Permanência para idosos (ILPI). Embora muitos idosos consigam viver e se manter sozinhos ou com ajuda de familiares, alguns necessitam de institucionalização. Deste modo, as ILPIs assumem a responsabilidade de cuidar quando o idoso perde seus vínculos com sua rede social, assistindo ou dando suporte às suas necessidades, com a finalidade de melhorar sua saúde e qualidade de vida (BESSA MEP, et al, 2012). 
Com o envelhecimento populacional e o aumento da expectativa de vida, foi possível verificar mudanças no perfil epidemiológico da população, com o predomínio das doenças crônicas não transmissíveis (DCNT), entre as quais, a depressão, que é a desordem mais comum nessa faixa etária (LEITE VMM, et al., 2006; CAMPOLINA AG, et al., 2013).

A institucionalização faz com o que o idoso, muitas vezes, vivencie perdas em vários aspectos da vida, aumentando a vulnerabilidade a quadros depressivos que podem levar a quadros psiquiátricos, perda da autonomia e agravamento dos quadros patológicos preexistentes (CARREIRA L, et al., 2011).

Esse isolamento social leva o idoso a um estado de solidão, perda de liberdade, de autoestima, e muitas vezes perda da identidade e até a rejeição da própria vida, justificando a alta ocorrência de doenças mentais nos asilos (NÓBREGA IRAP, et al., 2015).

A prevalência de sintomas depressivos é maior em idosos institucionalizados do que os que moram com suas famílias (VIEIRA SKSF, et al., 2017). A depressão, sendo uma das doenças de maior prevalência entre os idosos, causa impactos negativos na sua vida, possivelmente sendo a causa mais comum de diminuição na qualidade de vida nessa população (SANTOS KT, et al., 2012).

Estudos realizados em diferentes países, observaram prevalências de sintomas depressivos que variaram entre 6,1\% (REPPERMUND S, et al., 2011) até 36,5\% (CASTRO C, et al., 2008). Já em idosos institucionalizados, essa prevalência é um pouco mais elevada, variando de 47\% (VAZ SFA e GASPAR NMS, 2011) a 49\% (SILVA C, et al., 2012).

Entre os indivíduos mais suscetíveis à ocorrência de depressão estão as mulheres, os idosos com situação econômica desfavorável, aqueles com história de eventos estressores na vida, idosos com percepção de baixa qualidade de vida e condições de saúde, aqueles com alguma limitação funcional, e os acometidos por doenças crônicas e dor (BLAY SL, et al., 2007; GUIMARÃES LA et al., 2019).

Além dos sintomas depressivos devemos considerar outras consequências para a saúde e a qualidade de vida dos idosos como declínio da capacidade funcional, limitação na realização de atividade física, diminuição da mobilidade, isolamento social e perda da autonomia e da independência para a execução das atividades de vida diária (AVD) (BALTES PB e SMITH J, 2006). De acordo com a Classificação Internacional de Funcionalidade (CIF), o termo funcionalidade abrange as funções e estruturas do corpo; atividade e participação social, e fatores ambientais. Refere-se à capacidade de a pessoa cuidar de si mesma e de desempenhar tarefas e papéis sociais (WHO, 2001; FARIAS N e BUCHALLA CM, 2005).

Com o avançar da idade, as perdas funcionais tornam-se mais evidentes e o idoso deixa de realizar atividades básicas de vida diária, diminuindo assim sua capacidade funcional. Em virtude do crescimento exponencial de idosos no país, a preocupação em relação à capacidade funcional tem aumentado em diversos setores, pois o conhecimento dos fatores que afetam a funcionalidade dos idosos é fundamental para o planejamento e realização de medidas de promoção e prevenção da saúde (VEIGA B, et al., 2016).

Diante disso, a avaliação da capacidade funcional tornou-se indispensável para a escolha da intervenção mais adequada e monitorização da situação clínica funcional dos idosos, visando a promoção de um envelhecimento saudável e uma melhor qualidade de vida (CASAGRANDE GHJ, et al., 2013). Diante do exposto, o objetivo do presente estudo foi avaliar a prevalência de sintomas depressivos e a capacidade funcional em idosos institucionalizados.

\section{MÉTODOS}

Trata-se de um estudo do tipo observacional transversal. Utilizou-se a técnica não probabilística, para selecionar os sujeitos que participaram do estudo. Os critérios de inclusão dos sujeitos foram: ter 60 anos de idade ou mais, residir em uma ILPI do município, concordância em participar do estudo e não apresentar comprometimento grave de linguagem ou compreensão que pudesse interferir na veracidade das respostas. A amostra foi posteriormente estratificada quanto as variáveis sociodemográficas, nível de incapacidade, 
presença ou não de sintomas depressivos e demais variáveis pertinentes ao estudo (Hipertensão arterial e Diabetes Mellitus).

Foram encontrados 108 idosos residentes nas três Instituições analisadas. Destes, 58 idosos foram excluídos por não atenderem os critérios de inclusão, 15 por problemas psiquiátricos, 28 por não atingirem a pontuação mínima no Mini Mental, 17 por se recusarem a assinar o TCLE. Foram sujeitos da pesquisa 50 idosos, sendo 13 homens e 37 mulheres.

A coleta de dados teve início após a leitura e assinatura do Termo de Consentimento Livre e Esclarecido (TCLE) e consistiu em entrevista estruturada e aplicação de questionários com os idosos institucionalizados. Ocorreu entre os meses de setembro e outubro de 2018, por meio de entrevistas individuais com os idosos nas instalações das ILPI.

Os idosos institucionalizados responderam um questionário, onde foram verificados os aspectos demográficos e socioeconômicos.

Para avaliar o estado cognitivo foi utilizado o Mini Exame do Estado Mental, para a detecção dos sintomas depressivos foi utilizada a Escala de depressão geriátrica e para avaliar a funcionalidade foi utilizado o World Health Organization Disability Assessment Schedule (WHODAS 2.0).

Os dados foram tabulados e analisados no software estatístico (Statistical Package for Social Sciences SPSS 13.0® (Chicago, IL, USA). Os dados numéricos foram apresentados como média e desvio padrão e os dados categóricos foram descritos como número absoluto e porcentagem total. A associação entre sintomas depressivos, funcionalidade, dados sociodemográficos e condições de saúde foi estimada pelo teste qui-quadrado de Pearson.

O nível de significância estatística foi definido em $5 \%$ para todos os testes $(p<0,05)$. A pesquisa foi aprovada pelo Comitê de Ética em Pesquisa da Universidade do Estado de Minas Gerais, (parecer no 3.040.675). Os participantes foram esclarecidos quanto aos objetivos da pesquisa bem como procedimentos aos quais foram submetidos e assinaram um termo de consentimento livre e esclarecido (TCLE). A participação dos idosos foi voluntária atendendo à Resolução 466/2012 do Conselho Nacional de Saúde.

\section{RESULTADOS E DISCUSSÃO}

Esse estudo foi realizado com uma amostra de 50 idosos institucionalizados em quatro ILPI da cidade de Divinópolis e foram distribuídos segundo as características sociodemográficas e frequência do resultado da escala de sintomas depressivos (EDG-15) (Tabela 1).

Quanto a caracterização dos participantes, a média de idade foi de 77,5 anos, variando de 60 a 99 anos, em uma amostra composta principalmente por mulheres (74\%), sem diferença significativa entre os sexos.

A predominante presença de mulheres idosas na presente pesquisa, corrobora com a denominada "feminização da velhice", fato crescente no Brasil, que é acompanhado por mudanças no perfil epidemiológico e assistencial (IBGE, 2009). No que se refere à escolaridade, $88 \%$ dos idosos não tinha ensino fundamental completo, sendo que $42 \%$ não foram a escola, $46 \%$ possuíam ensino fundamental incompleto, e apenas $12 \%$ possuíam ensino médio. Quanto ao estado civil, $48 \%$ eram solteiros, $34 \%$ viúvos, $16 \%$ separados e $2 \%$ casados.

A prevalência de sintomas depressivos nos idosos avaliados foi de $60 \%$, e destes, $44 \%$ apresentaram depressão leve/moderada e 16\% apresentaram depressão grave.

Destes pacientes com presença de sintomas depressivos, $34 \%$ se encontram com idade $\geq$ a 80 anos, $24 \%$ não possuem ensino fundamental completo, $30 \%$ não estudaram, $34 \%$ são solteiros, $36 \%$ tem uma qualidade de sono ruim, $24 \%$ apresentam incapacidade moderada e $18 \%$ apresentam incapacidade grave. De acordo com o Teste qui quadrado, há evidência de associação entre qualidade de sono e grau de depressão (sendo mais frequente em idosos com pior qualidade de sono $(p=0,01)$. 
Tabela 1 - Distribuição das características sociodemográficas e frequência do resultado da escala de sintomas depressivos (EDG-15).

\begin{tabular}{lccccc}
\hline \multicolumn{5}{c}{ EDG - 15 } \\
\hline \multirow{4}{*}{ Variável } & Distribuição & Sem & Depressão & Depressão & \\
& da & depressão & leve/moderada & grave & $P$ \\
& população & $\mathrm{N}(\%)$ & $\mathrm{N}(\%)$ & $\mathrm{N}(\%)$ & \\
& $\mathrm{N}(\%)$ & & & & \\
\hline
\end{tabular}

\begin{tabular}{|c|c|c|c|c|c|}
\hline Sexo & & & & & \\
\hline Masculino & $13(26,0)$ & $7(14,0)$ & $4(8)$ & $2(4)$ & \\
\hline & & & & & 0,47 \\
\hline Feminino & $37(74,0)$ & $13(26,0)$ & $18(36,0)$ & $6(12,0)$ & \\
\hline \multicolumn{6}{|l|}{ Idade } \\
\hline $60-69$ & $12(24,0)$ & $6(12,0)$ & $5(10,0)$ & $1(2)$ & \\
\hline $70-79$ & $13(26,0)$ & $6(12,0)$ & $2(4,0)$ & $5(10,0)$ & 0,34 \\
\hline$>80$ & $25(50,0)$ & $8(16,0)$ & $15(30,0)$ & $2(4,0)$ & \\
\hline \multicolumn{6}{|l|}{ Escolaridade } \\
\hline $\begin{array}{c}\text { Fundamental } \\
\text { incompleto }\end{array}$ & $23(46,0)$ & $11(22,0)$ & $10(20,0)$ & $2(4,0)$ & \\
\hline Médio & $6(12,0)$ & $3(6,0)$ & $2(4,0)$ & $1(2,0)$ & 0,56 \\
\hline Não estudou & $21(42,0)$ & $6(12,0)$ & $10(20,0)$ & $5(10,0)$ & \\
\hline \multicolumn{6}{|l|}{ Estado Civil } \\
\hline Solteiro & $24(48,0)$ & $7(14,0)$ & $13(26,0)$ & $4(8,0)$ & \\
\hline Casado & $1(2,0)$ & $0(00,0)$ & $0(00,0)$ & $1(2,0)$ & 0,23 \\
\hline Separado & $8(16,0)$ & $4(8,0)$ & $3(6,0)$ & $1(2,0)$ & \\
\hline Viúvo & $17(34,0)$ & $9(18,0)$ & $6(12,0)$ & $2(4,0)$ & \\
\hline \multicolumn{6}{|l|}{$\begin{array}{l}\text { Qualidade do } \\
\text { sono }\end{array}$} \\
\hline Ruim & $20(40,0)$ & $2(4,0)$ & $11(22,0)$ & $7(14,0)$ & $0,01^{*}$ \\
\hline Bom & $30(60,0)$ & $18(36,0)$ & $11(22,0)$ & $1(2,0)$ & \\
\hline
\end{tabular}

Legenda: *Valores de $p$ calculados pelo Teste Qui-Quadrado.

Fonte: Perina KCB, et al., 2020.

Foi possível observar que $32 \%$ dos idosos apresentam Diabetes Mellitus, e destes, $24 \%$ apresentam sintomas depressivos; e $64 \%$ dos idosos são hipertensos, e destes, 40\% apresentam sintomas depressivos (Tabela 2).

Utilizando o critério de categorização da variável incapacidade funcional em cinco níveis, os seguintes pontos de corte foram definidos: escore zero (nenhuma incapacidade funcional); escores 1 a 12 (incapacidade leve); escores 13 a 22 (incapacidade moderada); escores 23 a 36 (incapacidade grave) 37 a 48 (incapacidade extrema). Com relação à funcionalidade, a média total de acordo com o WHODAS 2.0 foi 
de 17,7 sendo os escores de incapacidade funcional maiores em mulheres, na faixa etária maior que 80 anos e nos idosos que apresentavam menor escolaridade. Dos pacientes avaliados, $2 \%$ não apresentavam incapacidade funcional, $32 \%$ apresentavam incapacidade leve, 36,0\% apresentavam incapacidade moderada, $26 \%$ apresentavam incapacidade grave e $4 \%$ apresentavam incapacidade extrema.

Foi possível observar que, dos 50 participantes estudados, 98\% (49) apresentaram algum grau de incapacidade funcional. Entre os seis domínios de incapacidade avaliados, os mais comprometidos foram, cognição, mobilidade e atividades de vida diária.

Tabela 2 - Distribuição das características antropométricas, morbidade e frequência do resultado da escala de sintomas depressivos (EDG-15).

\begin{tabular}{|c|c|c|c|c|c|}
\hline \multicolumn{6}{|c|}{ EDG-15 } \\
\hline Variável & $\begin{array}{l}\text { Distribuição da } \\
\text { população } \\
\text { n (\%) }\end{array}$ & $\begin{array}{c}\text { Sem depressão } \\
n(\%)\end{array}$ & $\begin{array}{c}\text { Depressão } \\
\text { leve/moderada } \\
\mathrm{n}(\%)\end{array}$ & $\begin{array}{l}\text { Depressão } \\
\text { grave } \\
\text { n (\%) }\end{array}$ & $P$ \\
\hline \multicolumn{6}{|l|}{ Hipertensão } \\
\hline Sim & $32(64,0)$ & $12(24,0)$ & $13(26,0)$ & $7(14,0)$ & \multirow{2}{*}{0,35} \\
\hline Não & $18(36,0)$ & $8(16,0)$ & $9(18,0)$ & $1(2,0)$ & \\
\hline \multicolumn{6}{|l|}{$\begin{array}{c}\text { Diabetes } \\
\text { Mellitus }\end{array}$} \\
\hline Sim & $16(32,0)$ & $4(8,0)$ & $9(18,0)$ & $3(6,0)$ & \multirow{2}{*}{0,33} \\
\hline Não & $34(68,0)$ & $16(32,0)$ & $13(26,0)$ & $5(10,0)$ & \\
\hline \multicolumn{6}{|l|}{ WHODAS } \\
\hline $\begin{array}{l}\text { Nenhuma } \\
\text { dificuldade }\end{array}$ & $1(2,0)$ & $1(2,0)$ & $0(0,0)$ & $0(0,0)$ & \multirow{5}{*}{0,50} \\
\hline $\begin{array}{l}\text { Dificuldade } \\
\text { Leve }\end{array}$ & $16(32,0)$ & $9(18,0)$ & $6(12,0)$ & $1(2,0)$ & \\
\hline $\begin{array}{l}\text { Dificuldade } \\
\text { Moderada }\end{array}$ & $18(36,0)$ & $6(12,0)$ & $8(16,0)$ & $4(8,0)$ & \\
\hline $\begin{array}{l}\text { Dificuldade } \\
\text { Grave }\end{array}$ & $13(26,0)$ & $4(8,0)$ & $6(12,0)$ & $3(6,0)$ & \\
\hline $\begin{array}{l}\text { Dificuldade } \\
\text { Extrema }\end{array}$ & $2(4,0)$ & $0(0,0)$ & $2(2,0)$ & $0(0,0)$ & \\
\hline
\end{tabular}

Legenda: Valores de $p$ calculados pelo Teste Qui-quadrado.

Fonte: Perina KCB, et al., 2020.

Na população de estudo foi possível observar que aproximadamente $60 \%$ dos idosos investigados tiveram alteração na EDG-15, com presença de sintomas depressivos. Esses achados corroboram com os estudos de Matias AGC, et al. (2016) e Guimarães LA, et al. (2019), que demonstraram uma prevalência de sintomas depressivos em idosos institucionalizados de $52,6 \%$ e $54,8 \%$, respectivamente. Segundo Nogueira LE, et al. (2014), a depressão não deve ser considerada apenas um estado de tristeza ou algo relacionado apenas ao envelhecimento, pois é um transtorno afetivo complexo que provoca diversas alterações, traduzindo-se, portanto, como um problema de saúde pública.

Esses sintomas podem estar relacionados à perda, por parte do idoso, do seu direito de escolha, de ter que seguir uma rotina de horários, à sensação de não ser mais importante, à aflição que o idoso enfrenta em conviver com o desconhecido, perda de privacidade, dificuldade de criar vínculos e superar perdas (BRASIL, 2007).

Ao nível da população idosa e tendo por base a amostra constituída por idosos institucionalizados de ambos os sexos, confirma-se a maior prevalência, embora sem significância estatística, de sintomas 
depressivos em idosos do sexo feminino (48\%). Nogueira LE, et al. (2014) e Guimarães LA, et al. (2019), também relatam maior prevalência de sintomas depressivos em idosos do sexo feminino, $35,9 \%$ e $64,7 \%$, respectivamente, corroborando com os resultados apresentados.

Dados da literatura, como os observados por Hellwig N, et al. (2016), sugerem maior vulnerabilidade das mulheres ao desenvolvimento de sintomas depressivos. Dentre as possíveis explicações está a maior suscetibilidade aos eventos estressores determinados por papéis sociais e de gênero, privação de estrogênio e o fato de que as mulheres vivem, em média, mais do que os homens e essa idade mais avançada vem acompanhada por uma maior incidência de doenças crônicas, entre elas, a depressão. Moura MAV, et al. (2010), afirmam que a atenção à saúde da mulher idosa deve ser uma preocupação constante dos profissionais da saúde, considerando suas necessidades físicas e emocionais, a fim de melhorar a qualidade de vida. Dessa forma, novas demandas surgem especialmente no tocante às especificidades do planejamento do cuidado à saúde da mulher idosa.

A população da presente pesquisa foi composta em sua maioria por idosos que apresentavam baixa ou nenhuma escolaridade, $88 \%$, e destes, $54 \%$ apresentavam sintomas depressivos. Segundo Madeira CST, et al. (2013), esse fato pode ser explicado como reflexo da sociedade tradicional dos séculos passados, em que não havia cobrança com relação aos estudos, pois o importante era trabalhar em função da família. De acordo com os dados do IBGE (2009), mesmo com as atuais oportunidades de alfabetização, ainda são escassos no Brasil idosos que possuem um maior grau de escolaridade.

No que se refere ao estado civil, foi possível observar que a maioria dos idosos eram solteiros e viúvos, $48 \%$ e $34 \%$, respectivamente, e destes, 34\% e 16\% apresentavam sintomas depressivos. Lini EV, et al. (2016) citam a falta de companheiro e ausência de filhos como um dos fatores que predispõem a institucionalização de idosos. Em seu estudo, 90,5\% dos idosos institucionalizados não possuíam companheiro. Segundo eles, por tradição histórica, os companheiros mostram-se como principais cuidadores em domicílio. E quando os idosos vivem sozinhos e se deparam com algumas incapacidades funcionais, enfrentam dificuldades em receber o cuidado em domicílio e acabam por necessitar de institucionalização. Gullich I, et al. (2016) também observaram em seu estudo, maior número de sintomas depressivos em idosos solteiros (34\%), justificado pelo fato de que, idosos solteiros que vivem quase sempre sozinhos, sem ninguém para confiar e compartilhar experiências, manifestam maior suscetibilidade para a sintomatologia depressiva, corroborando com a presente pesquisa,

Os resultados do estudo mostraram associações importantes entre a qualidade do sono e sintomas depressivos $(p=0,01)$. Com relação aos idosos que apresentavam sintomas depressivos, $36 \%$ apresentavam qualidade de sono ruim. Guimarães LA, et al. (2019) também encontraram uma associação significativa entre sintomas depressivos e qualidade do sono, corroborando com a presente pesquisa. Cardoso DCM (2013) observou que quanto mais grave os sintomas depressivos, maior a dificuldade de pegar no sono $(p=0,21)$, maior o número de vezes que se acorda a noite $(p=0,14)$ e pior a qualidade de sono ( $p=0,23$ ). Segundo Guimarães LA, et al. (2019), a má qualidade do sono pode levar a prejuízos na saúde e dificuldade nas atividades de vida diária do idoso. Consequentemente, os distúrbios de sono vêm acompanhados de problemas de memória, atenção, concentração e déficit de atenção, sendo estes sintomas muitas vezes confundidos nesses idosos como demência e déficit cognitivo.

A prevalência de incapacidade funcional na presente pesquisa, para pelo menos uma atividade, foi de $98 \%$, onde a maioria (36\%), apresentava incapacidade funcional moderada. Destes, $24 \%$ apresentavam sintomas depressivos, sem associação significativa entre eles. Segundo Nóbrega IRAP, et al. (2015), a baixa capacidade funcional ou incapacidade funcional estão diretamente relacionadas com a presença de depressão. Segundo os autores, idosos com depressão apresentam maiores comprometimentos físicos, sociais e funcionais, interferindo na qualidade de vida com consequente redução ou perda da independência funcional.

Com relação à funcionalidade, a média total de acordo com o WHODAS 2.0 foi de 17,7, sendo os escores de incapacidade funcional maiores em idosos com idade igual ou superior a 80 anos (50\%). 
Segundo Guimarães LA, et al. (2019), os sintomas depressivos nos idosos podem levar a comprometimentos funcionais, tornando-os mais dependente na realização de suas atividades cotidianas. Duca GFD, et al. (2012) verificaram maior número de institucionalização em idosos que possuíam incapacidade de executar suas atividades de vida diária e que possuíam idade igual ou superior a 80 anos, corroborando com a presente pesquisa.

Com relação aos seis domínios do WHODAS 2.0 que foram avaliados, o mais comprometido foi a cognição. Segundo Souza VL, et al. (2009), a institucionalização interfere no desempenho cognitivo quando comparados com idosos que residem em suas casas. Em uma revisão realizada por Nóbrega IRAP, et al. (2015), quatro artigos relacionaram a depressão a um pior estado cognitivo. Segundo eles, a grande frequência de queixas cognitivas levou à criação do termo pseudodemência depressiva. $O$ segundo item que teve índice mais elevado de acordo com o WHODAS 2.0 foi à mobilidade. Veiga B, et al. (2016) explicam tal acontecimento pelo fato de o idoso perder ou diminuir seus papéis ocupacionais. Já o terceiro item foram os domínios nas atividades de vida diária. Contrariando nossos achados, Souza AAD, et al. (2018), observaram que maior parte de sua amostra se encontrava funcional para as atividades básicas de vida diária.

Foi possível observar neste estudo uma proporção elevada de portadores de doenças crônicas não transmissíveis, fatos confirmados pelo processo de transição epidemiológica no Brasil. A morbidade mais comum encontrada nos idosos foi a Hipertensão arterial (64\%) seguido da Diabetes Mellitus (32\%). Tanto a Hipertensão arterial quanto a Diabetes Mellitus foi mais comum em mulheres, com $54 \%$ e $26 \%$, respectivamente. Pereira DS, et al. (2015) também encontraram em seu estudo uma alta prevalência de doenças crônicas não transmissíveis, sendo $46,2 \%$ dos idosos com Hipertensão Arterial e $18 \%$ dos idosos com Diabetes Mellitus.

Dos pacientes com hipertensão (64\%) e Diabetes Mellitus (32\%), 40\% e 24\%, respectivamente, apresentam sintomas depressivos, sem associação entre eles. Já Nóbrega IRAP, et al. (2015) afirmam que a associação entre doenças crônicas e sintomas depressivos é bimodal, de modo que a depressão pode levar ao surgimento de doenças crônicas ou estas podem aumentar o risco de depressão em idosos. Segundo Mesquita JS, et al. (2016), os idosos precisam de maiores cuidados específicos, para isso é necessário ações inovadoras, reorganizando o serviço de saúde com uma abordagem que associe o crescente envelhecimento da população e ações voltadas a mudança demográfica e epidemiológica.

\section{CONCLUSÃO}

Foi possível concluir que a prevalência de sintomas depressivos e incapacidade funcional em idosos institucionalizados foi de $60 \%$ e $98 \%$, respectivamente. Ressalta-se que a detecção precoce dos sintomas depressivos é importante para evitar o desenvolvimento do quadro depressivo, prevenindo, desta forma, seus efeitos negativos para a saúde e qualidade de vida desses idosos. Vale também destacar a importância dos resultados obtidos para o profissional Fisioterapeuta junto à equipe de saúde, pois ao adquirir conhecimento sobre o processo de envelhecimento e as doenças que podem acometer o idoso, ele se torna mais atento, no sentido de identificar as necessidades do idoso, reduzindo suas dificuldades e melhorando sua qualidade de vida.

\section{REFERÊNCIAS}

1. ALVES DB e BARBOSA MTS. Desigualdades na Mortalidade por doenças Crônicas entre idosos e sua Associação com Indicadores Socioeconômicos no Brasil. Revista Brasileira de Ciências do Envelhecimento Humano, 2010; 7(1): 22-33.

2. BALTES PB e SMITH J. Novas fronteiras para o futuro do envelhecimento: a velhice bem-sucedida do idoso jovem aos dilemas da quarta idade. A Terceira Idade, 2006; 17(36): 7-31.

3. BESSA MEP, et al. Idosas residentes em instituições de longa permanência: uso dos espaços na construção do cotidiano. Acta Paulista de Enfermagem, 2012; 25(2): 177-182. 
4. BLAY SL, et al. Depression Morbidity in Later Life: Prevalence and Correlates in a Developing Country. The American journal of geriatric psychiatry: official journal of the American Association for Geriatric Psychiatry, 2007; 15(9): 790-9.

5. BRASIL. Ministério da Saúde (MS). Envelhecimento e saúde da pessoa idosa. Brasília: MS; 2007.

6. CAMPOLINA AG, et al. A Transição de Saúde e as Mudanças na expectativa de Vida Saudável da População Idosa: Possíveis Impactos da Prevenção de Doenças Crônicas. Cadernos de Saúde Pública, 2013; 29(6): 12171229.

7. CARDOSO DCM. Sono, Depressão e Afetividade em Idosos Institucionalizados. Dissertação de mestrado, Coimbra, 2013.

8. CARREIRA L, et al. Prevalência de depressão em idosos institucionalizados. Revista de Enfermagem, 2011; 19(2): 268-273.

9. CARVALHO JAM e RODRÍGUEZ-WONG LL. A Transição da Estrutura etária da População Brasileira na primeira metade do Século XXI. Cadernos de Saúde Pública, 2008; 24(3): 597-605.

10. CASAGRANDE GHJ, et al. Qualidade de vida e incidência de depressão em idosas que frequentam grupos de terceira idade. Revista Brasileira de ciências do Envelhecimento Humano, 2013; 10(1): 52-65.

11. CASTRO C, et al. Factors associated with Depressive Symptoms Measured by the 12-Item General Health Questionnaire in Community-Dwelling Older Adults. Revista Brasileira de Psiquiatria, 2008; 30(2): 104-109.

12. DUCA GFD, et al. Indicadores da institucionalização de idosos: estudo de caso e controles. Revista de Saúde Pública, 2012; 46(1): 147-153.

13. FARIAS N e BUCHALLA CM. A classificação internacional de funcionalidade, incapacidade e saúde da organização mundial da saúde: conceitos, usos e perspectivas. Revista Brasileira de Epidemiologia, São Paulo, 2005; 8(2): 187193.

14. GUIMARÃES LA, et al. Sintomas depressivos e fatores associados em idosos residentes. Ciências e Saúde Coletiva, 2019; 24(9): 3275-3282.

15. GULLICH I, et al. Depressão entre idosos: um estudo de base populacional no sul do brasil. Revista Brasileira de epidemiologia, 2016; 19(4): 691-701.

16. HELLWIG N, et al. Sintomas depressivos em idosos: estudo transversal de base populacional. Saúde Pública, 2016; 21(11): 3575-3584.

17. IBGE - Projeção da população do Brasil: população brasileira envelhece em ritmo acelerado. Rio de Janeiro: IBGE, 2008.

18. IBGE - Instituto Brasileiro de Geografia e estatística. Sobre a condição de saúde dos idosos: indicadores selecionados. Indicadores Sociodemográficos e de Saúde no Brasil. Brasília (DF): IBGE, 2009.

19. LEITE VMM, et al. Depressão e envelhecimento: Estudo nos participantes do Programa Universidade Aberto à terceira Idade. Revista Brasileira de Saúde Materno Infantil, 2006; 6(1): 31-38.

20. LINI EV, PORTELLA MR, DORING M. Fatores associados a institucionalização de idosos: estudo caso-controle. Revista Brasileira de Geriatria e Gerontologia, 2016; 19(6): 1004-1014.

21. MACIEL MG. Atividade física e funcionalidade do idoso. Motriz: Revista de Educação Física, 2010; 16(4): 1024-32.

22. MADEIRA CST, et al. Depressão em idosos hipertensos e diabéticos no contexto da atenção primária em saúde. Revista APS, 2013; 16(4): 393-398.

23. MATIAS AGC, et al. Indicadores de depressão em idosos e os diferentes métodos de rastreamento. Einstein, 2016; 14(1): 6-11.

24. MESQUITA JS, et al. Promoção da saúde e integralidade na atenção ao idoso: uma realidade brasileira? Revista Kairós Gerontologia, 2016; 19(1): 227-238.

25. MORAES EN. atenção a saúde do idoso: Aspectos Conceituais. Organização Pan-Americana da saúde, 2012; 1(1): 102.

26. MOURA MAV, et al. A qualidade na atenção a saúde da mulher idosa: um relato de experiência. Escola Anna Nery, 2010; 14(4): 848-855.

27. NÓBREGA IRAP, et al. Fatores associados à depressão em idosos institucionalizados: revisão integrativa. Saúde debate, 2015; 39(105): 536-550.

28. NOGUEIRA LE, et al. Rastreamento de sintomas depressivos em idosos na EstratégiaSaúde da Família. Revista de Saúde Pública, 2014; 48(3): 3608-377.

29. PEREIRA DS, et al. Qualidade de vida e situação de saúde de idosos: um estudo de base populacional no Sertão Central do Ceará. Revista Brasileira de Geriatria e Gerontologia, 2015; 18(4): 893-908.

30. REPPERMUND S, et al. The Relationship of Current Depressive Symptoms and Past Depression with Cognitive Impairment and Instrumental Activities of Daily Living in an Elderly Population: The Sydney Memory and Ageing Study. Journal of Psychiatric Research, 2011; 45(12): 1600-1607.

31. SANTIAGO LM e MATTOS IE. Sintomas depressivos em idosos institucionalizados. Revista de Saúde Pública, 2014; 48(2): 216-224.

32. SANTOS KT, et al. Depressive Symptoms and Motor Performance in the Elderly: A Population based Study. Brazilian Journal of Physical Therapy, 2012; 16(4): 295-300.

33. SILVA C, et al. Cross-Cultural adaptation of the World Health Organization Disability Assessment Schedule (WHODAS 2.0) into Portuguese. Revista da Associação Médica Brasileira, 2012; 59: 234-240.

34. SOUZA VL, et al. Perfil das habilidades cognitivas no envelhecimento normal. Revista CEFAC, 2009; 12(2): 186192. 
35. SOUZA WC, et al. Exercício físico na promoção da saúde na terceira idade. Saúde Meio Ambiente, 2015; 4(1): 5565.

36. SOUZA AAD, et al. Qualidade de vida e incapacidade funcional entre idosos cadastrados na estratégia de saúde da família. ABCS Health Sciences, 2018; 43(1): 14-24.

37. VAZ SFA e GASPAR NMS. Depressão Em Idosos Institucionalizados No Distrito de Bragança. Revista de Enfermagem Referência, v. III série, 2011; 4: 49-58.

38. VEIGA B, et al. Avaliação de funcionalidade, incapacidade de idosos longevos em acompanhamento ambulatorial utilizando o WHODAS 2.0. Revista Brasileira de Geriatria e Gerontologia, 2016; 19(6): 1015-1021.

39. VIEIRA SKSF, et al. Características sociodemográficas e morbidades entre idosos institucionalizados sem declínio cognitivo. Revista de pesquisa cuidado é fundamemtal, 2017; 9(4): 1132-1138.

40. WORLD HEALTH ORGANIZATION (WHO). The International Classification of Functioning. Disability and Health, Geneva, 2001. 